\title{
The Drosophila Shark tyrosine kinase is required for embryonic dorsal closure
}

\author{
Rafael Fernandez, ${ }^{1}$ Fumitaka Takahashi, ${ }^{1}$ Zhao Liu, ${ }^{3}$ Ruth Steward, ${ }^{3}$ David Stein, ${ }^{1,2}$ \\ and E. Richard Stanley ${ }^{1,4}$
}

Departments of ${ }^{1}$ Developmental and Molecular Biology and ${ }^{2}$ Molecular Genetics, Albert Einstein College of Medicine, The Bronx, New York 10461 USA; ${ }^{3}$ Waksman Institute, Department of Molecular Biology and Biochemistry, Rutgers University, Piscataway, New Jersey 08854 USA

\begin{abstract}
Dorsal closure (DC) in the Drosophila embryo requires the coordinated interaction of two different functional domains of the epidermal cell layer-the leading edge (LE) and the lateral epidermis. In response to activation of a conserved c-Jun amino-terminal kinase (JNK) signaling module, the dorsal-most layer of cells, which constitute the LE of the stretching epithelial sheet, secrete Dpp, a member of the TGF $\beta$ superfamily. Dpp and other LE cell-derived signaling molecules stimulate the bilateral dorsal elongation of cells of the dorsolateral epidermis over the underlaying amnioserosa and the eventual fusion of their LEs along the dorsal midline. We have found that flies bearing a Shark tyrosine kinase gene mutation, shark ${ }^{1}$, exhibit a DC-defective phenotype. Dpp fails to be expressed in shark ${ }^{1}$ mutant LE cells. Consistent with these observations, epidermal-specific reconstitution of shark function or overexpression of an activated form of c-Jun in the shark ${ }^{1}$ mutant background, rescues the DC defect. Thus, Shark regulates the JNK signaling pathway leading to Dpp expression in LE cells. Furthermore, constitutive activation of the Dpp pathway throughout the epidermis fails to rescue the shark ${ }^{1}$ DC defect, suggesting that Shark may function in additional pathways in the LE and/or lateral epithelium.
\end{abstract}

[Key Words: Dorsal closure; Shark tyrosine kinase; epithelial sheets; Drosophila embryogenesis; Jun kinase]

Received September 16, 1999; revised version accepted January 30, 2000.

The synchronized movement of epithelial cell layers is crucial for the development of the body plan in metazoans. Folding, invagination, stretching and fusion of epithelia are accompanied by changes in cell shape, and the coordination of these changes involves interactions among the extracellular matrix, the cell surface, and the cytoskeleton (for review, see Leptin 1994). In the Drosophila embryo, gastrulation is followed by retraction of the germ band, which leaves the dorsal-most portion of the embryo covered by the extraembryonic membrane, termed the amnioserosa. The cells of the ectoderm, which give rise to the larval epidermis, cover the ventral and lateral portions of the embryo. In a process known as dorsal closure $(\mathrm{DC})$, the cells from both sides of the ectoderm migrate over the underlying amnioserosa leading to the enclosure of the embryonic viscera. Movement of these epithelial layers depends exclusively on cell elongation and does not require mitotic growth or cell recruitment (for review, see Martinez-Arias 1993). DC has been divided into three phases (for review, see Knust 1997). Initially, the dorsal-most cells of the lateral epi-

\footnotetext{
${ }^{1}$ Present address: Howard Hughes Medical Institute, University of Pennsylvania Medical School, 415 Curie Boulevard, CRB 320, Philadelphia, PA 19104.

${ }^{4}$ Corresponding author.

E-MAIL rstanley@aecom.yu.edu; FAX (718) 430-8567.
}

dermis, which form the leading edge (LE) of the epithelial sheet, elongate along the dorsal-ventral axis and accumulate actin and nonmuscle myosin in their dorsal edges. Second, the more lateral epidermal cells lose their polygonal shape and also elongate, thereby moving the whole epidermis dorsally. Finally, the LE cells of both sides arrive at the dorsal midline and fuse. DC-defective mutations affect one or more steps of the ectodermal layer migration process, resulting in lack of cuticle secretion in the dorsal region, a characteristic dorsal open phenotype, and embryonic lethality (for review, see Martinez-Arias 1993).

The cellular events that characterize DC in Drosophila, along with an extensive collection of dorsal open mutations, make this an ideal system for analyzing the mechanisms orchestrating the movement of epithelial sheets. The recent molecular characterization of several of these loci has shed light on some of the signaling events involved in ectodermal cell stretching. Ectodermal expression of the TGF $\beta$ homolog, decapentaplegic $(d p p)$, by cells of the LE is essential to promote migration of the lateral ectoderm (Riesgo-Escovar and Hafen 1997b). This domain of Dpp expression depends on activation of a Ser/Thr kinase cascade of members of the stress-activated protein kinase (SAPK)/Jun amino-terminal kinase (JNK) pathway (for review, see Kyriakis and 
Avruch 1996). Epistatic analysis and biochemical data indicate that misshapen (msn, a Ste-20-related kinase) functions upstream of hemipterous (hep, a MKK4-7 homolog) (Glise et al. 1995; Su et al. 1998). hep, in turn, activates basket (bsk, a homolog of JNK) (Glise et al. 1995; Riesgo-Escovar et al. 1996; Sluss et al. 1996). Phosphorylation of a fly Jun homolog (D-jun) by Bsk leads to the activation of an AP-1 type complex, presumably formed by D-Jun and D-Fos [kayak (kay)], which ultimately leads to transcriptional activation of Dpp only in cells of the LE (Riesgo-Escovar and Hafen 1997a; for review, see Noselli 1998; Noselli and Agnes 1999).

Movement of the epithelial sheet behind the LE depends on the receptor for Dpp encoded by the genes thick veins $(t k v)$ and punt (put). In contrast to mutants of the JNK pathway, in which stretching of all cells of the ectoderm fails, cells of the LE undergo normal elongation in $t k v$ and put mutants with no elongation of lateral cells (Riesgo-Escovar and Hafen 1997b). All components of the Dpp signaling pathway are also important for DC as they also show a dorsal open phenotype on sensitized genetic backgrounds (Chen et al. 1998). However, whether their role is to directly regulate lateral epidermal stretching or to promote the patterning of lateral cells, rendering them competent to receive additional cues that lead to their elongation toward the dorsal midline has yet to be determined (for review, see Leptin 1994).

The identification of several of the DC-defective loci has outlined a JNK-Dpp-Tkv/Put, LE to lateral ectoderm pathway. However, cloning and biochemical analysis of additional DC loci points to greater complexity in these signaling processes. For example, a JNK-independent pathway in the LE has been implicated in studies with Pkn, a Drosophila homolog of the putative Rho/ Rac effector target family of serine/threonine kinases (Lu and Settleman 1999). In addition, kay activity is not only required in the LE but also in the lateral ectoderm, and expression of Kay by cells of the lateral ectoderm depends on Tkv-Put activity (Riesgo-Escovar and Hafen 1997b). It was also shown recently that the DC mutant myoblast city (mbc, Erickson et al. 1997) does not affect Dpp expression at the LE. Mbc is a homolog of the Racspecific activator and integrin signaling protein DOCK180 (Kiyokawa et al. 1998; Nolan et al. 1998). Because $\mathrm{Mbc}$ is expressed in the lateral ectoderm and not in the amnioserosa (Erickson et al. 1997), Mbc function is likely to be required only in the lateral ectoderm. These findings suggest either the existence of additional inputs that work in conjunction with Tkv-Put to contribute to the stretching of the lateral ectoderm in Drosophila embryos or novel mechanisms of signaling through Dpp receptors.

Recently we have described a Drosophila nonreceptor tyrosine kinase, termed Shark $\underline{\mathrm{SH} 2}$ domain ankyrin $\underline{\text { re}}-$ peat kinase, Ferrante et al. 1995), which contains from amino to carboxyl terminus, a Src homology 2 (SH2) domain (N-SH2), five ankyrin repeats, a second $\mathrm{SH} 2$ domain (C-SH2), a proline-rich and basic region, and a tyrosine kinase domain. This study addresses the in vivo role of Shark in Drosophila development. Analysis of the phenotypes associated with a shark loss-of-function mutation demonstrate that Shark activity is essential for the migration of the dorsolateral epidermis of the embryo. We show that the Shark kinase functions in DC upstream of Dpp expression by LE cells.

\section{Results}

\section{Isolation of a mutation in shark}

shark was mapped previously by in situ hybridization to polytene segment 53A1 (Ferrante et al. 1995) on the right arm of the second chromosome. Further molecular and genetic mapping has localized the shark locus to the 52F interval (Fig. 1a). The shark gene was found to be contained in a P1 clone (DS06638), cytologically mapped by the Berkeley Genome Project (www.fruitfly.org), to 52F2-52F8. In addition, independently mapped YAC clones were used to further confirm the chromosomal location. Clones DY506 (52F-53C) and DY664 (52E153A2), but not DYR15-76 (52D7-52E9), were shown by PCR and Southern blot analysis to contain the entire shark gene. In situ hybridization to polytene chromosomes with the P1 clone DS06638 indicates that shark is removed by deficiencies $D f(2 R) I p 7, I p 8$, and $I p 4$ but not by $I p 1$, defining the position for the shark locus to a genetic interval between the proximal breakpoint of $D f(2 R) J p 8$ and the distal breakpoint of DfJp(2R)P4 (Fig. 1a).

A search for recessive lethal mutations in the region uncovered by $D f(2 R) J p 8$ identified 20 lethal complementation groups. Of these, 12 complementation groups were removed by $D f(2 R) J p 4$ as well and represented candidate mutations in the shark locus. To determine if shark was represented by one of these lethal complementation groups and thereby encodes an essential function, we introduced transgenes expressing shark cDNA under the control of a heat shock promoter, into flies mutant for each complementation group in trans to the deficiency. The lethality of the single mutant allele of complementation group W-4 was the only phenotype rescued when shark expression was induced by heat shock treatment. This was true for either of the two hs-shark transgenic lines, and we therefore renamed the rescued mutation $\operatorname{shark}^{1}$ (Table 1). Moreover, a hs-shark transgene encoding a catalytically inactive form (K698R) of Shark failed to rescue shark ${ }^{1}$ mutants, indicating that the Shark tyrosine kinase activity is required to rescue the mutant phenotype (data not shown).

Analysis of the lethal phase of the single shark mutant recovered indicated that either shark ${ }^{1}$ homozygotes, or shark $^{1} / D f(2 R) I p 7$ or shark ${ }^{1} / D f(2 R) I p 4$ trans-heterozygotes, resulted in pupal lethality (data not shown). Sequencing of shark ${ }^{1}$ revealed the introduction of a stop codon at position 210 that predicts a protein containing only the first 210 amino acids of the shark ORF, which is likely to be nonfunctional, as two ankyrin repeats and the C-SH2, the proline-rich, and the tyrosine kinase domains are deleted completely. The truncation of the 
Figure 1. Schematic representation of the shark locus mapping and basis of the shark $^{1}$ mutation. (a) shark locus mapping. A chromosomal in situ hybridization, in which a P1 clone (DS06638) containing the shark gene was used to probe a pair of homologs of the genotype $D f(2 R) J p 7 /+$, is shown at the top, aligned with a drawing of the banding pattern of this region (Lindsley and Zimm 1992). The hybridization signal appears only on the wild-type chromosome. Molecular mapping of the shark gene to Drosophila genomic P1 (DS06638) and YAC (DY506, DY664, DYR15-76) clones was done by PCR analysis to test for shark coding sequences, and Southern blot analysis to establish presence of the entire shark gene. The cytological boundaries of the deficiency chromosomes $D f(2 R) I p 1, D f(2 R) I p 4$, $D f(2 R) I p 7$, and $D f(2 R) I p 8$, which we confirmed or rectified by viability over cytologically single P-element lethal insertions from the Berkeley Genome Project, are shown below the genomic clones. The shaded region highlights the shark mutant group (W-4), lined up to the deficiency chromosomes and genomic clones. (b) Representation of the wildtype and shark ${ }^{1}$ ORFs. The shark ${ }^{1}$ locus encodes a truncated form of Shark protein due to a $\mathrm{C} \rightarrow \mathrm{T}$ transition at nucleotide 685 that changes Gln-210 to a stop codon.

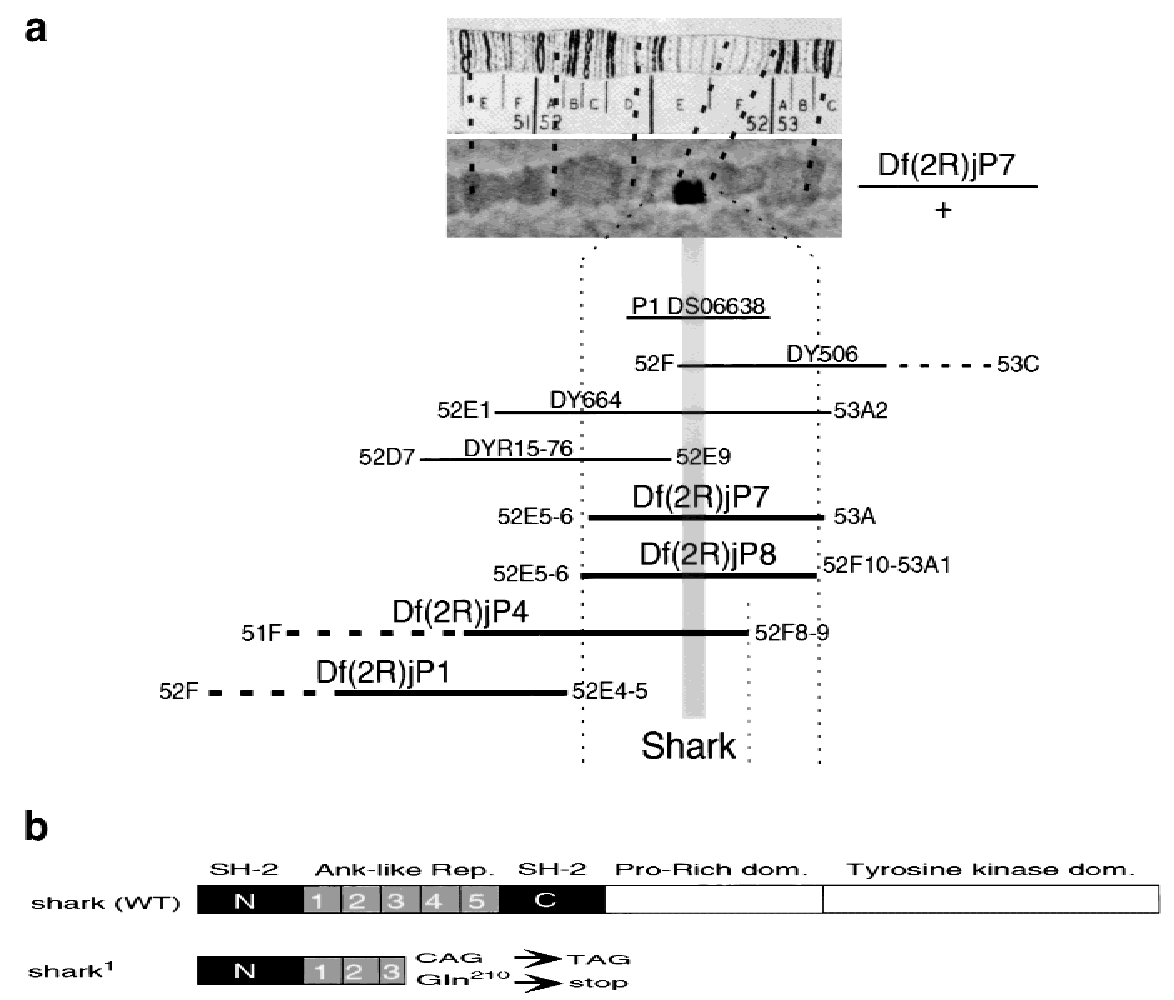

shark ORF encoded by this mutant allele confirms the identity of the W-4 complementation group as the shark locus (Fig. 1b).

\section{shark mRNA is expressed maternally}

To localize the distribution of shark transcripts, we performed whole-mount in situ hybridization in wild-type embryos. Large amounts of shark mRNA were observed in ovarian nurse cells (not shown), unfertilized eggs, and syncitial blastoderm embryos (Fig. 2A). Owing to the abundant maternal contribution, shark message expression appears ubiquitous during early embryogenesis up until about stages 6-7 (stages according to Campos-Ortega and Hartenstein 1985). By the end of germ-band extension, shark mRNA is expressed primarily in the ectoderm. Following germ-band retraction, stages 12-15, the strongest shark mRNA expression occurs uniformly in the epidermis and other ectodermally derived structures such as the anterior foregut, hindgut, and Malphi-

Table 1. Heat shock-dependent rescue activity of the lethality of shark ${ }^{1} / \mathrm{Df}(2 \mathrm{R}) \mathrm{Jp} 4$ flies by hs-shark-10 and hs-shark-2b transgenes

\begin{tabular}{|c|c|c|c|}
\hline \multirow{2}{*}{$\begin{array}{l}\text { Maternal genotype of } D f(2 R) / p 4 \\
\text { to test rescue activity }\end{array}$} & \multicolumn{3}{|c|}{ Percentage of $\operatorname{shark}^{1} / D f(2 R) I p 4$ adults recovered $\mathrm{at}^{\mathrm{b}}$} \\
\hline & $22^{\circ} \mathrm{C}$ & $27^{\circ} \mathrm{C}$ & $27^{\circ} \mathrm{C}(+7 \mathrm{HS})^{\mathrm{c}}$ \\
\hline w; hs-shark-2B; Df(2R)Ip4/CyO & $20 / 350(6 \%)^{\mathrm{d}}$ & $45 / 336(13 \%)$ & $80 / 234(34 \%)$ \\
\hline w; Df(2R)Ip4/CyO; hs-shark-10 & $30 / 400(8 \%)$ & $50 / 340(15 \%)$ & $81 / 230(35 \%)$ \\
\hline
\end{tabular}

a Fly stocks were generated in which a balanced $w ; D f(2 R) / p 4 / C y O$ was placed in combination with two independent transgenic lines (hs-shark-2B and hs-shark-10, mapped to the X and third chromosomes, respectively) expressing a full-length shark cDNA under control of the heat shock promoter (hs-Casper, Thummel et al. 1988). At least one allele of the 12 complementation groups mapped to the deficiency interval that contains shark (see text and Fig. 1a) was test-crossed to these hs-shark deficiency stock combinations to examine the ability of hs-shark-2B and -10 to rescue the lethality of any of the combinations $1(2 R) W 1-12 / D f(2 R) J p 4$.

${ }^{\mathrm{b}} \mathrm{The}$ number of $\mathrm{CyO}^{+}\left[\operatorname{shark}^{1} / D f(2 R) J p 4\right]$ over the total number of flies; corresponding percentage in brackets.

${ }^{\mathrm{c}}(\mathrm{HS})$ Heat shock treatment $\left(1 \mathrm{hr}\right.$ at $\left.37^{\circ} \mathrm{C}\right)$.

${ }^{\mathrm{d}}$ Under conditions resulting in minimal rescue, shark ${ }^{1} / D f(2 R) I p 4$ flies showed hep-like defects with variable penetrance ranging from $40 \%$ to $80 \%$ (see Fig. 3). 


\section{in situ hybridization}
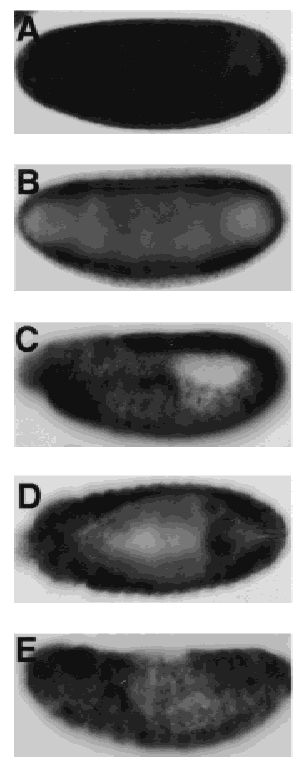

anti-Shark (N-SH2)
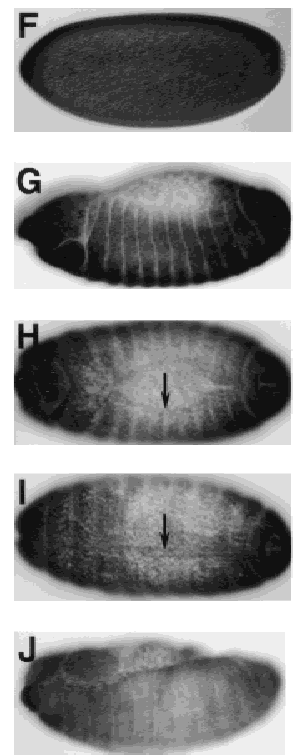

immunostaining
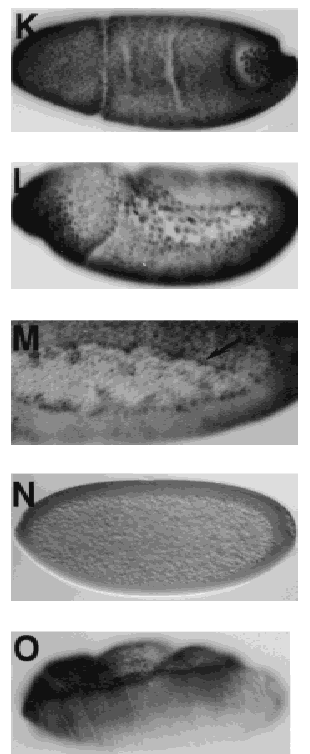

Figure 2. Expression patterns of shark mRNA $(A-$ $E)$ and protein $(F-I, K-M)$ in wild-type embryos. $(A-E)$ Whole mount in situ hybridizations with a fulllength shark antisense (digoxin-labeled) riboprobe. Oriented, anterior to the left, are syncitial $(A$, stages 2-3) and cellular ( $B$, stages 4-5) blastoderm embryos showing large amounts of shark mRNA (lateral views). (C) Lateral view of a germ-band-extended embryo (stage 9) showing ectodermal staining. $(D)$ Dorsal view of stage 13 embryo. shark mRNA expression is seen in the epidermis, hindgut, and Malphigian tubules. By stages 15-16 (E, lateral view), shark mRNA levels have decreased significantly. (FO) Shark protein expression in wild-type $(F-I, K-M)$ and shark ${ }^{1}$ GLC $(J, N, O)$ embryos, detected with antiserum raised against a GST fusion protein containing the Shark N-SH2 domain. Ubiquitous Shark protein in cellular blastoderm $(F$, stage 4$)$ and at the onset of gastrulation $(K)$. Protein expression becomes more restricted during germ-band elongation ( $L$, stages 8-10, lateral view). Protein expression at stages $13-14$ is primarily epidermal $(G)$. Shark protein expression at the LE (arrows) of the epidermis at stage $10(M$, lateral view, high magnification), stage $14(H$, dorsal view $)$, and on completion of DC at stage $15(I$, dorsal view). Staining is reduced in early $(N)$, mid $(J)$, and late $(O)$ stage embryos derived from shark ${ }^{1}$ GLCs (zygotic genotype shark ${ }^{1} / \operatorname{shark}^{1}$ ).

gian tubules. Most of the shark message is down-regulated by the end of embryogenesis (Fig. 2A-E). Protein expression was analyzed using an antiserum raised to the Shark N-SH2 domain. In general, staining with this antiserum paralleled the expression of shark mRNA (Fig. $2 \mathrm{~F}-\mathrm{I}, \mathrm{K}-\mathrm{M})$. Shark protein is expressed abundantly from the onset of cellularization, resulting in ubiquitous expression until the commencement of gastrulation. From this stage on, its expression is most prominent in the ectoderm and some of the ectodermally derived tissues. At stage 10 (full germ-band extension) and thereafter, Shark protein expression increases again but is restricted to the ectoderm, foregut, hindgut, and Malphigian tubules. During germ-band extension (stages 9-10) and in migrating epidermal cells during DC (stage 13-14), Shark protein expression is particularly strong at the LE of the epidermis. By the end of embryogenesis, epidermal expression decreases. Embryos homozygous for deficiency $D f(2 R) I p 7$, which removes the shark locus, as well as shark ${ }^{1}$ homozygote embryos, show a reduction in immunoreactivity only after stages 13-14 (data not shown), consistent with a substantial contribution of maternal mRNA. In contrast, staining is reduced in embryos derived from shark ${ }^{1}$ germ-line clones (GLCs; see below) (Fig. 2N,J,O), consistent with the specificity of the antiserum for Shark, which could detect residual mutant protein. Also, in agreement with genetic evidence for a predominant role of Shark in the epidermis (see below), late shark ${ }^{1}$ GLC-derived embryos consistently lack all immunoreactivity in epidermal cells (Fig. 2O). This pattern of Shark protein expression differed significantly from the previously reported immunolocalization of Shark protein with antibodies directed to the kinase domain (Ferrante et al. 1995), where poor pregastrulation and epidermal staining was seen that was contributed to by the dominance of apical staining of epithelia and staining of the tracheal system by one of three antibodies in the mixture of antipeptide antibodies used. This antibody was subsequently shown to detect an unrelated epitope (R. Fernandez, unpubl.).

Rescue of a shark mutation suggests that shark functions in the INK pathway

Wild-type hs-shark transgene rescue experiments without heat shock provided suboptimal rescue conditions (defined as those that yield progeny below the $33 \%$ of adults expected for a fully viable genotype, Table 1), which often gave rise to flies with a split thorax and/or hep-like defects (Fig. 3a). This indicates that rescue of lethality can occur at levels of Shark that are insufficient to support normal thoracic closure. These phenotypes also point to a role for Shark in processes in which the Jun kinase kinase hep (Glise et al. 1995; Glise and Noselli 1997) and the fos homolog kay (Zeitlinger et al. 1997; Riesgo-Escovar and Hafen 1997a) have been shown to be involved. Both hep and kay have been shown to be components of the JNK signaling module, which regulates cell elongation in epithelial cell sheets undergoing morphogenetic stretching (Noselli 1998). In addition to the split thorax defects, rescued shark ${ }^{1} / D f(2 R) J p 4$; hsshark females fertilized with sperm carrying the shark ${ }^{1}$ allele gave rise to embryos that showed a DC-defective phenotype (Fig. 3b). Like the split thorax phenotype, defects in embryonic DC have also been associated with loss of function in components of the JNK pathway that regulates $d p p$ expression at the LE of the embryonic epi- 
Figure 3. Phenotypes of the shark ${ }^{1}$ mutation: $|a|$ Adult phenotype of partially rescued flies. Dorsal view of the thorax of flies (anterior is left) of the following genotypes: (A) w; shark ${ }^{1} / D f(2 R) J p 4$; hs-shark-10/hsshark-10 showing fully rescued flies, indistinguishable from wild type; $(B) \mathrm{W}$; hs-shark-2B; $\operatorname{shark}^{1} / D f(2 R) I p 4$ flies showing mild defects due to incomplete rescue. A streak of naked cuticle is evident, marking the thoracic midline (arrow); $(C, D)$ W; hs-shark-2B; $\operatorname{shark}^{1}$ / $D f(2 R) I p 4$, under conditions of minimal rescue activity (no heat shock) showing a severe split thorax phenotype. The arrow points to a cleft running along the dorsal thoracic midline that results from incomplete movement toward the midline of both imaginal wing disc epithelia. The arrowhead in $D$ points to the remnant of the left wing that failed to outgrow (hep-like defect; Riesgo-Escovar and Hafen 1997a; Zeitlinger et al. 1997). (b) Lethality and dorsal open phenotype of shark $^{1}$ embryos derived from hs-shark-10 rescued $\operatorname{shark}^{1} / D f(2 R) I p 4$ females. (A) Cuticular pattern (lateral view) of a wild-type embryo; $(B)$ mild cuticle defects (anterior open phenotype); $(C)$ the more prevalent, strong dorsal open phenotype of embryos derived from rescued shark ${ }^{1}$ females of the indicated genotype.

dermis (Riesgo-Escovar and Hafen 1997b; Zeitlinger et al. 1997; Zeitlinger and Bohmann 1999).

\section{shark ${ }^{1}$ homozygote germ-line clones exhibit a strong} DC-defective phenotype

To investigate the phenotypic effects of the loss of Shark function, we eliminated maternal Shark activity by generating shark ${ }^{1}$ homozygote GLCs /Chou and Perrimon 1996). Zygotes derived from GLCs with the shark ${ }^{1}$ mutation show a marked reduction in reactivity (Fig. $2 \mathrm{~N}, \mathrm{~J}, \mathrm{O})$ in anti-Shark immunostaining. Because shark ${ }^{1}$ is predicted to encode a truncated polypeptide containing the entire N-SH2 domain, which was the immunogen used to raise the antiserum in these experiments, the reduced immunoreactivity with the anti-GST/N-SH2 serum suggests that this allele produces a mutant protein that is expressed at low levels. This result is consistent with Western blot analysis of balanced shark ${ }^{1}$ mutant embryos and adults, which also show low levels of the truncated protein predicted to be encoded by shark ${ }^{1}$ (data not shown).

Analysis of cuticles derived from shark ${ }^{1}$ homozygote GLCs fertilized with males of the genotypes shark ${ }^{1} /$ CyO or $D f(2 R) I p 4 / C y O$ (Fig. 4a) demonstrates that loss of maternal and zygotic Shark activity yields a strong DC-defective phenotype, similar to embryos produced by partially rescued shark ${ }^{1} / D f(2 R) J p 4$; hs-shark flies. Moreover, shark ${ }^{1}$ GLCs fertilized with sperm carrying either the shark ${ }^{1}$ allele or $D f(2 R) I p 7$ give identical phenotypes, providing genetic evidence that shark ${ }^{1}$ is a null mutation. Finally, zygotic rescue of all cuticular defects via the paternal allele, which leads to the eventual production of viable, fertile adults, indicates that Shark activity is not required for early embryonic events such as organization of the cellular blastoderm or gastrulation.

\section{Shark participates in signaling in LE cells}

Cuticles of maternal and zygotic shark null embryos are identical to those of strong alleles or maternal and zygotic loss-of-function alleles in hep, msn, bsk, kay, and $D$-jun mutants, among others. Cellular analysis of the epidermal cells in this group of mutants has revealed that the defect in cuticle secretion on the dorsal-anterior side, together with the failure to enclose the embryonic gut, is due to a defect in the directional elongation of the cells of the LE and lateral epidermis (Noselli 1998). Immunostaining of epidermal cells with monoclonal antibodies to the band 4.1 homolog protein Coracle (Fehon et al. 1994) reveals that shark ${ }^{1}$ has the same effect on epidermal cell function and epithelial cell morphogenesis (Fig. 4b, A-D) as all other members of the JNK pathway. Both LE and lateral epidermal cells fail to stretch toward the dorsal midline over the anterior three-quarters of the embryo, consistent with the deficient cuticle secretion in the anterior-dorsal region.

This defect, evident in all epidermal cells, demonstrates a requirement of Shark for the elongation of LE cells and suggests a role for Shark in the regulation of Dpp transcription, or the production of other signals activated in LE cells that subsequently target elongation of adjacent lateral cells. Because Shark is expressed throughout the epidermis, it is possible that it also has an active role in the lateral cells, operating downstream or in parallel to the Dpp/Tkv-Put pathway (Riesgo-Escovar and Hafen 1997b). To investigate these possibilities, we analyzed Dpp expression by in situ hybridization in shark ${ }^{1}$ null embryos and studied the effects of reconstitution of the JNK and the Dpp pathways, as well as Shark function, in a shark ${ }^{1}$ null background by using a combination of upstream activating sequence (UAS) transgenes and Gal4 driver lines specific for the LE region and lateral cells of the embryonic epidermis. 


\section{a}

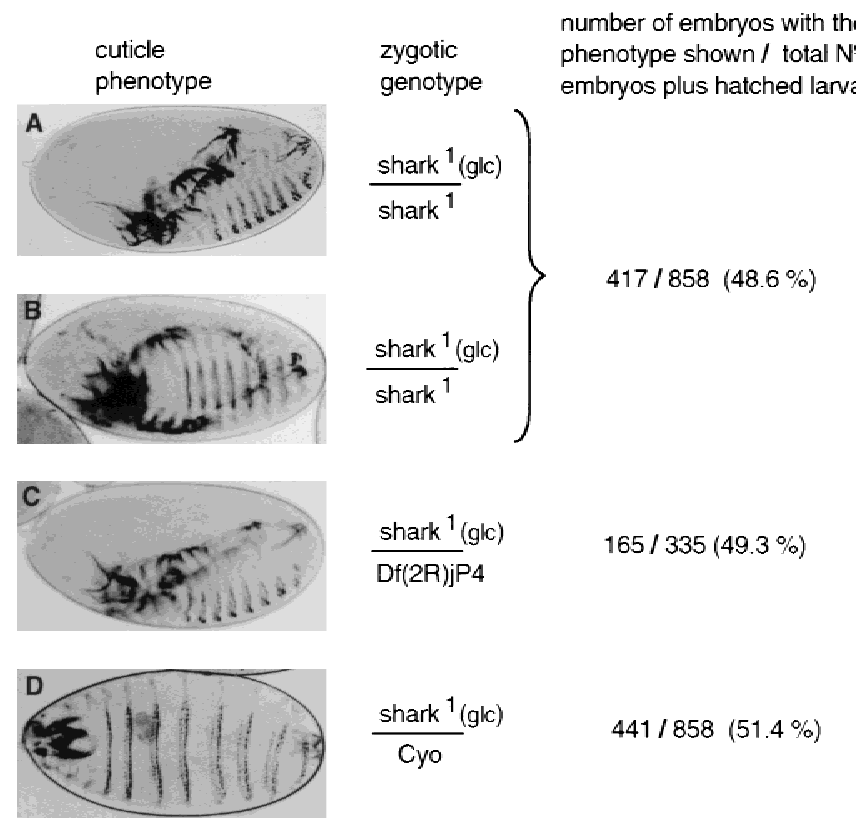

b

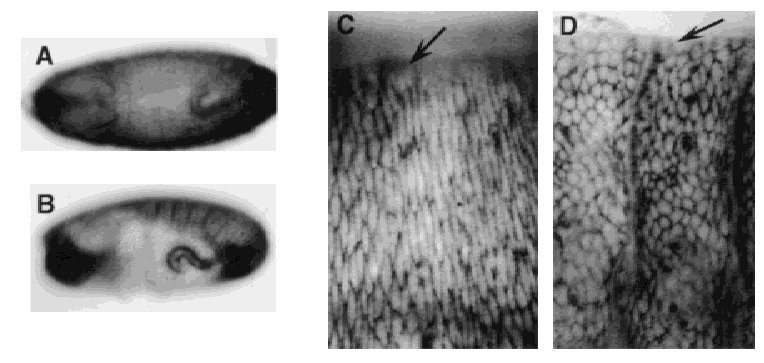

C

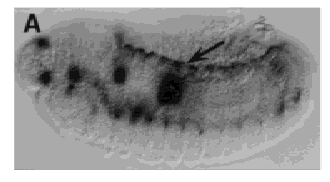

Figure 4. Phenotypic analysis of shark ${ }^{1}$ embryos lacking maternal and zygotic Shark activity. (a) Cuticular phenotypes associated with complete loss of shark function (anterior to the left). $(A, B)$ Lateral and ventral views, respectively, of representative cuticles derived from shark ${ }^{1}$ GLCs fertilized with shark ${ }^{1}$ chromosomes and showing a strong dorsal open phenotype. [In this experiment females of the genotype FRT42B, ovo ${ }^{1} / F R T 42 B$, shark ${ }^{1}$ were crossed to shark ${ }^{1}$ CyO-balanced males, giving rise to two different zygotic genotypes: $\operatorname{shark}^{1}(\mathrm{glc}) / \operatorname{shark}^{1}$ (maternally derived chromosome/paternally-derived chromosome), with the embryonic lethal phenotype shown in $A$ and $B$ and their fully paternally rescued siblings, shark ${ }^{1}($ glc) $/ C y O$, shown in $D]$. (C) shark ${ }^{1}$ GLCs fertilized with $D f(2 R) I p 7$ chromosomes show phenotypes indistinguishable from those in $A$ and $B$, indicating that shark ${ }^{1}$ is a null mutation and that this phenotype is not due to any second site mutations in cis to shark ${ }^{1}$. [In this case, females of the genotype FRT42B, ovoD1/FRT42B, shark $^{1}$ were crossed to $D f(2 R) I p 7 / C y O$-balanced males, yielding two different zygotic genotypes: shark ${ }^{1}(\mathrm{glc}) / D f(2 R) / p 7$, with phenotypes indistinguishable from those in $A$ and $B$ and the paternally rescued siblings, shark $\left.{ }^{1}(\mathrm{glc}) / C y O\right]$. (D) The lethality of shark ${ }^{1}$ GLCs fertilized by a shark ${ }^{1}$-containing chromosome can be fully rescued by a wild-type paternal chromosome (a $C y O$ balancer), which yields fertile adults. $(b)$ Epidermal cell morphogenesis and $d p p$ expression in wild-type $(A, C)$ and shark ${ }^{1}$ mutant $(B, D)$ embryos undergoing DC. The overall epidermal morphology is shown in whole-mount embryos $(A, B)$, and the shape of epidermal cells, revealed by mAb anti-coracle antibody immunostaining in a wild-type embryo (lateral view, $C$ ) shows normal stretching in the direction of the dorsal midline. (D) Lateral view of a shark ${ }^{1}$ GLC that fails to complete DC showing that LE (arrows) and lateral epidermal cells maintain their polygonal shape and fail to elongate toward the dorsal midline. (c) Stage 14 embryos showing whole-mount in situ hybridizations to a $d p p$ antisense riboprobe expression, in a paternally rescued shark ${ }^{1}$ GLC $(A)$, which appears indistinguishable from wild type. $(B)$ A shark $^{1}$ GLC fertilized with a $D f(2 R) I p 7$ chromosome lacks expression of $d p p$ message only at the LE. In both cases, the LE of the epidermis is indicated by an arrow.

A comparison of the embryonic $d p p$ mRNA expression by whole-mount in situ hybridization, between wildtype and shark ${ }^{1}$ GLCs, showed that Shark is required for Dpp expression by cells of the LE following germ-band retraction. From stage 12 on, Dpp expression is absent in LE cells of shark ${ }^{1}$ GLCs (Fig. 4c), whereas expression in the ventral cord and midgut, which occurs in a JNKindependent manner (Noselli 1998), remains unaffected.

Consistent with the Shark expression pattern, the shark ${ }^{1}$ DC phenotype was completely rescued by expression of UAS-shark under control of the 69BGal4 driver, which expresses ubiquitously throughout the ectoderm (Fig. 5A,B). Interestingly, however, expression of the LE region driver PnrGal4 in the same UAS-shark line failed to rescue the shark ${ }^{1}$ DC defect (data not shown). This result, with a driver that expresses in three to five rows of the most dorsal ectodermal cells (Heitzler et al. 1996), suggests that either Shark expression is also required in the lateral epidermis or that Shark is required for PnrGal4 expression in the LE region.

Because no obvious genetic interactions were obtained between Shark and mutations of the JNK pathway (data not shown), we tested whether constitutive activation of the JNK or the Dpp pathway could rescue the shark ${ }^{1}$ DC phenotype (Fig. 5C-J). When shark ${ }^{1}$ GLCs were generated in the background of flies carrying a shark ${ }^{1}$ chromosome with an inserted transposon expressing an activated form of c-Jun (hs-SEjunAsp; Treier et al. 1995), shark ${ }^{1}$ DC defects were completely rescued, in some cases, as determined by the decreased penetrance of embryonic lethality $(\sim 10 \%$ lower than the fully penetrant $50 \%$ observed without the expression of hs-SEjunAsp and by the complete or partial enclosure observed in unhatched embryos (Fig. 5C-F). These results are consis- 
Figure 5. Effect of domain specific expression of Shark, activated c-Jun or activated Tkv on rescue of the dorsal open cuticle phenotype in shark ${ }^{1} \mathrm{mu}$ tant embryos. $(A, B)$ Embryos derived from shark ${ }^{1}$ GLC fertilized with sperm carrying the shark ${ }^{1}$ allele, expressing UAS-shark and 69B-Gal4 show complete rescue of the dorsal open phenotype. Furthermore, these embryos develop normally to fertile adults (data not shown). (C-F) Embryos derived from shark ${ }^{1}$ GLCs fertilized with sperm carrying the shark ${ }^{1}$ allele, and expressing an activated form of c-Jun under control of the heat shock promoter (hs-SEJunAsp). Partial rescue $(C, D)$ or complete rescue $(E, F)$ are readily observed and account for $\sim 35 \%$ of the shark null embryos (the percentage of severe DC-defective embryos is reduced to $\sim 15 \%$ ), when embryos are heat-shocked between 5 and 10 hr after egg laying. $(G, H)$ Embryos from shark ${ }^{1}$ GLCs fertilized with a $\mathrm{CyO}$ balancer (paternally rescued) and carrying UAS-Tkv* and 69B-Gal4 show partially dorsalized cuticles that are indistinguishable from those seen in wild-type embryos expressing UAS-Tkv* and 69B-Gal4. $(I, J)$ Embryos derived from shark ${ }^{1}$ GLC fertilized with sperm carrying the shark ${ }^{1}$ allele, expressing UAS-Tkv* and 69B-Gal4 show partial dorsalization (lack of
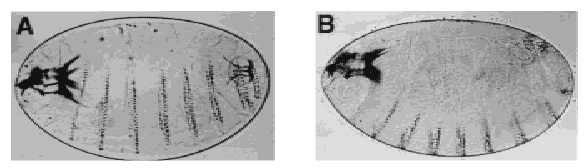
$\frac{+}{\text { UAS-shark }^{\prime}} ;$ shark $^{1}$ (glc) $; \frac{69 \mathrm{~B}-\text { Gal4 }}{+}$
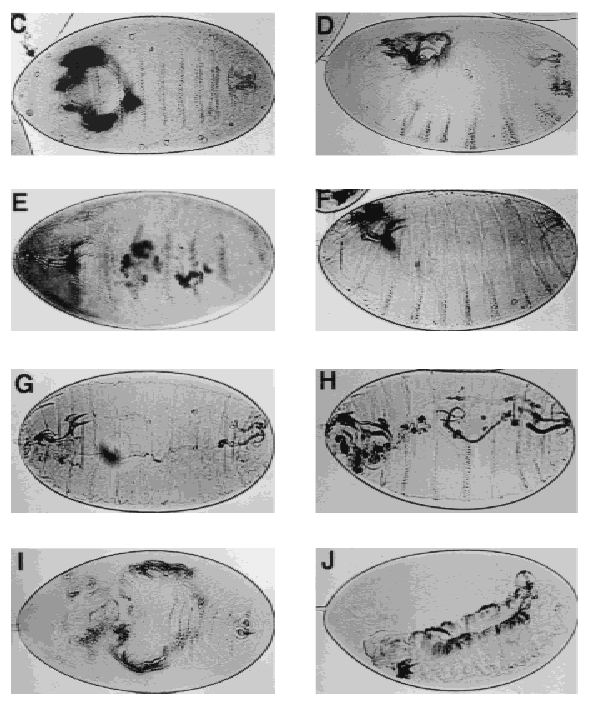

ventral denticle belts) and no rescue of the dorsal open phenotype. The genotypes are written with the maternally derived chromosome on top [e.g., $\left.\operatorname{shark}^{1}(\mathrm{glc})\right]$ and the paternally derived chromosome below (e.g., shark ${ }^{1}$, hs-SEjunAsp). $(A, C, E)$ Ventral views; $(G, I)$ dorsal views; $(B, D, F, H, J)$ lateral views.

tent with the action of Shark upstream of bsk (JNK) in the JNK pathway in LE cells.

If Shark was required solely for Dpp expression in LE cells, expression of a constitutively activated $\mathrm{Tkv}$ receptor $\left(\mathrm{Tkv}^{\star}\right)$ under the control of the $69 \mathrm{~B}$ driver throughout the ectoderm should rescue the shark ${ }^{1}$ DC phenotype. Although embryos expressing the combinations of UAS$\mathrm{Tkv}^{\star}$ and 69B-Gal4 can be readily identified by the partial dorsalization of their cuticles (Fig. 5G-J), two distinctive kinds of partially dorsalized cuticles were observed: (1) those that should be expressing the combination of transgenes in a paternally rescued background and that showed complete enclosure $(\sim 12 \%)$, and (2) an equivalent percentage of embryos, showing marginal or no rescue of the DC defect, in spite of being dorsalized, demonstrating expression of both $\mathrm{Tkv}^{\star}$ and 69B-Gal4. Because msn and bsk mutations are rescued by 69B-Gal4-driven UAS-Tkv ${ }^{\star}$ (Riesgo-Escovar and Hafen 1997b; Su et al. 1998), these findings raise the possibility that Shark may also function in an additional signal generating pathway in LE cells, in the lateral epidermis, or both.

\section{Discussion}

We have described a mutation in the nonreceptor tyrosine kinase Shark and the characterization of the phenotypes observed in ectodermal epithelial layers caused by loss of Shark function. As shown by the Shark requirement for Dpp expression and the rescue with activated Jun, our results indicate that it regulates morphogenesis of the embryonic epidermis. Shark is essential for activation of the JNK pathway in LE cells.

shark loss of function results in phenotypes similar to a subset of defects associated with loss of Dpp signaling activity in migrating ectodermal epithelial layers

Temperature-dependent rescue of the lethality of shark mutants with heat shock-driven cDNA transgenes resulted in dramatic adult phenotypes that suggested a role for Shark in morphogenesis of ectodermal epithelia. Adult flies expressing insufficient levels of Shark exhibit a split thorax phenotype, indicating a role for Shark activity in the dorsal joining of the wing imaginal epithelia derived from the left and right wing discs during pupal development. Unilateral deletions of wings and a cleft along the middle of the dorsal thorax have been reported in homozygous escapers of weak hep alleles (Glise et al. 1995) and partially rescued kay mutants (Riesgo-Escovar and Hafen 1997a; Zeitlinger et al. 1997). As both hep and kay are components of the JNK/Dpp/Tkv-Put pathway, shown to be essential for epidermal cell sheet elongation and fusion during embryonic DC (Noselli 1998), it was proposed that wing disc epithelial stretching may be regulated by a JNK/Dpp/Tkv-Pnt pathway similar to the one operating in DC (Riesgo-Escovar and Hafen 1997a). Recently, Zeitlinger and Bohmann (1999) have formally demonstrated that thorax closure is controlled by the same molecular events that regulate DC, in which the JNK pathway promotes LE expression of Dpp and Puck- 
ered. Consistent with this, mutations that affect Dpp signaling show a split thorax and/or hep-type defect(s) under a variety of hypomorphic conditions that yield adult flies. For example, this is the case in viable $t k v$ (Terracol and Lengyel 1994), mad (Raftery et al. 1995), and med (Hudson et al. 1998) heteroallelic combinations, as well as in $t k v 6 / t k v 6$ adults, whose phenotypes have been enhanced by loss of function in one copy of the TGF $\beta$-like 60A locus [also known as glass bottom boat $(g b b)]$, mad, med, or put (Chen et al. 1998).

Adult shark ${ }^{1}$ rescued females also showed reduced Shark accumulation in developing eggs as evidenced by the production of shark ${ }^{1}$ homozygous embryos with strong DC zygotic defects. Considering that homozygous shark ${ }^{1}$ mutants die as pupae and the early preblastoderm expression of shark mRNA is high, it seems plausible that the maternally derived Shark activity is sufficient to facilitate embryonic development. The requirement of Shark for DC was ultimately confirmed by removing Shark activity through the generation of shark mutant GLCs. Even though the shark mutant phenotypes that we have described are consistent with loss of Dpp expression and/or failure of signaling by Dpp receptors, unlike some other mutations in these pathways- $d p p$, $t k v$, put, mad, and med - which affect pattern formation and cell determination during eye, wing, and leg imaginal disc development (Raftery and Sutherland 1999), the shark $^{1}$ mutation does not seem to affect the development of imaginal disc-derived structures, aside from dorsal fusion of the thorax. That is, typical rough eye phenotypes, abnormalities in wing vein patterning, lack of distal tarsal segments in the adult legs, as well as dorsoventral patterning defects in the embryo, were not observed in shark ${ }^{1}$ mutants. Thus, as with other loci controlling the epidermal LE-specific JNK pathway (Noselli 1998), shark ${ }^{1}$ phenotypes are clearly restricted to cell migration and the stretching of ectodermal cell sheets. However, it remains possible that further reduction in Shark expression, below levels in the developing shark ${ }^{1}$ pupae, would lead to effects in other structures.

\section{Shark activity is required in LE cells of ectodermal cell layers undergoing Dpp-dependent migration and stretching}

To date, two functionally different cell types have been defined among the ectodermal cells of the embryo that eventually differentiate into cuticle-secreting epidermis: (1) LE cells, and (2) the cells of the lateral epidermis. In response to extracellular cues believed to emanate from the underlying amnioserosa, ectodermal LE cells are specifically induced to secrete Dpp, from mid- to late-embryogenesis. LE expression of Dpp is not only essential to coordinate developmental processes within the ectoderm itself but also to promote patterning of the juxtaposing dorsal mesoderm (Xu et al. 1998). In addition to their ability to transduce a signaling cascade mediated by a SAPK/JNK cascade that leads to Dpp expression, the morphology of the LE cells is dramatically altered following germ-band retraction, when the LE cells elongate along the dorso-ventral axis as they migrate in the direction of the dorsal midline (Noselli 1998). Dpp secretion by LE cells triggers a response in the immediately adjacent cells of the epidermis, which results in the concerted elongation of the entire epidermis along the dorso-ventral axis and enclosure of the dorsal side of the embryo. This response in lateral epidermal cells is transduced by the type I and II heterodimeric Dpp receptors Tkv and Put. Analysis of the effect of the combined loss of maternal and zygotic shark function, with markers that reveal changes in epidermal cell shape and function in the developing embryo, indicate that elongation of all cells of the epidermis is equally affected. This results in a strong DC-defective cuticular phenotype that is caused, at least in part, by loss of Dpp expression by LE cells, as revealed by Dpp in situ hybridization.

However, apart from its involvement upstream of the JNK pathway in LE cells, our transgenic rescue experiments suggest that Shark is either required in another pathway in the LE, in a pathway other than the Dpp pathway in the lateral epidermis, or both. Shark is expressed throughout the ectoderm, and expression of Shark driven by the 69B driver in both the LE and the lateral epidermis completely rescues the shark ${ }^{1}$ DC phenotype. Consistent with Shark regulation of JNK or JNKlike pathways, the shark ${ }^{1}$ DC phenotype is rescued by ubiquitous expression of activated c-Jun. The failure of 69B-Gal4-driven activated Tkv receptor to rescue the shark $^{1}$ DC phenotype is consistent with the possible involvement of Shark in novel pathways in the lateral epidermis, or for the generation of LE signals in addition to Dpp, for which evidence has been obtained recently (Lu and Settleman 1999). Additional investigation of these possibilities is warranted.

The current findings clearly indicate that Shark regulates Dpp expression in LE cells (Fig. 6). The discovery that Shark is a member of this pathway should assist in the identification of additional upstream components. Recent studies indicate that a Drosophila c-src homolog, Src42A, also functions upstream of the JNK module in DC (Tateno et al. 2000). Consistent with both observations, during DC, tyrosine phosphorylation is prominent at the dorsal border of LE cells and is also found along the cell peripheries in the lateral epidermis (Harden et al. 1996). We speculate that Shark is an upstream regulator of multiple Ser/Thr protein kinase cascades, which regulate different biological effects, and that Shark transduces a signal(s) that is diversified in a manner analogous to receptor tyrosine kinase-mediated signaling that promotes pleiotropic effects in a variety of cell types.

\section{Materials and methods}

Genetic protocols

To screen for shark mutants, an EMS mutagenic screen for point mutations that fail to complement the deficiencies $D f(2 R) J p 8$ and $D f(2 R) J p 4$ was performed, essentially as described by Lewis and Bacher (1968). A detailed description will be published elsewhere (Z. Lin and R. Steward, unpubl.). The cytological characterization of the deficiency chromosomes was 


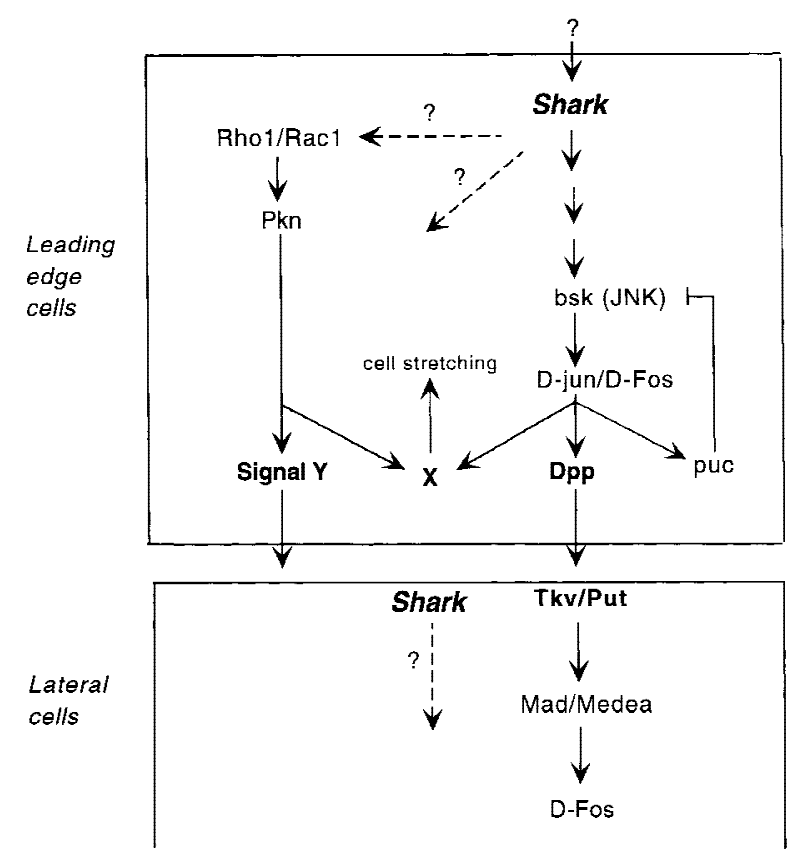

Figure 6. Schematic representation of Shark function in DC. The scheme summarizes our results, which indicate that Shark functions upstream of the JNK pathway in the LE, to regulate Dpp expression. The scheme also includes a JNK-independent pathway required in the LE that was proposed by Lu and Settleman (1999). Such a pathway might be a target for additional signals generated by Shark. In addition, Shark might also function in the lateral epidermis to regulate stretching of lateral epithelial cells (see text).

confirmed by testing their lethality in trans to single P-element lethal insertions mapped by the Berkeley Genome Project. To attempt P-element-mediated rescue of potential shark mutations, a full-length cDNA devoid of $5^{\prime}$ and $3^{\prime}$ UTRs was subcloned into the BgIII site of the transformation vector pCasperhs (Ashburner 1989). Two independent insertion lines, one on the X-chromosome (hs-shark-2B) and one on the third chromosome (hs-shark-10) were mated to a $w ; D f(2 R) I p 4 / C y O$ deficiency stock. Flies of the genotypes $w$; hs-shark-2B; $D f(2 R) I p 4 /$ CyO or w; $D f(2 R) I p 4 / C y O$; hs-shark-10 were crossed to alleles of each of the lethal complementation groups in the shark region to test for the ability of the transgene to complement. Rescue activity was assessed by scoring for survival of $\mathrm{CyO}^{+}$ flies from crosses that were performed at $25^{\circ} \mathrm{C}$, as well as crosses that were heat-shocked once a day for $1 \mathrm{hr}$ at $37^{\circ} \mathrm{C}$, from the first larval instar to late third larval instar stage of development.

To remove both maternal and zygotic Shark activity, GLC analyses were performed as described (Chou et al. 1993; Chou and Perrimon 1996). The shark ${ }^{1}$ allele was recombined into a chromosome carrying the $F R T 42 B\left(\mathrm{mw}^{+}\right)$transposon (Xu and Rubin 1993; Chou and Perrimon 1996). Heat shock (two 1-hr $37^{\circ} \mathrm{C}$ pulses at 48 and $72 \mathrm{hr}$ after egg laying)-mediated recombination and generation of GLCs was carried out by crossing females of the genotype hs-FLP7; FRT42B, $\operatorname{shark}^{1} / \mathrm{CyO}$ to $\mathrm{w}$; FRT42B, ovo ${ }^{D 1} / C y O$ males (Chou et al. 1993).

The heat shock and UAS-driven Shark expression plasmids used in rescue experiments were generated as follows: The shark ORF (only coding region) devoid of all UTR sequences was amplified by PCR using a shark cDNA (Ferrante et al. 1995) with the primers $5^{\prime}$-CCCAAAAAGGATCCGCGATAGTGAT-

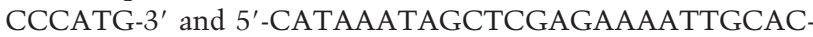
TCAGC-3'. PCR reactions were carried out with $P f u$ polymerase (STRATAGENE), digested with NcoI and NotI, and subcloned into the plasmid pBP4 (Driever et al. 1990). The insert was digested with $B g / I I$ and subcloned into the expression vectors pCaSpeR-hs and pUAST. Functionality of these constructs and the loss of catalytic activity of the K698R mutated shark constructs were confirmed by transient transfection into Schneider cells, followed by anti-Shark and anti-phosphotyrosine Western blotting. P-element-mediated transformation of hs-CaSpeR-Shark and pUAST-Shark was done according to Spradling and Rubin (1982).

Antibodies, immunostaining procedures, cuticle preparations, and photography

The antipeptide antibodies to the kinase domain of Shark have been described previously (Ferrante et al. 1995). Polyclonal antisera against a GST fusion protein (pGex-KG; Guan and Dixon 1991) containing the N-SH2 domain of the shark ORF (residues 9-119; Ferrante et al. 1995) were raised in rabbits (Cocalico Biologicals, Inc.) by injecting glutathione-agarose affinity-purified GST fusion protein (Smith and Johnson 1988). Antibodies to $\beta$-galactosidase, used to identify embryos that did not carry a balancer chromosome containing a lacZ transgene $(\mathrm{CyO}$, eve$l a c Z$, or $T M 6, T b, u b x-l a c Z$ ) were purchased from Sigma (mouse polyclonal, diluted 1:1000) or Cappel (rabbit polyclonal, diluted 1:3000). Anti-Coracle mAbs C566.9 and C615.16 were generously provided by Richard Fehon (Duke University, Durham, $\mathrm{NC)}$, and were used together at a 1:400 dilution. Anti-Shark $\mathrm{N}-\mathrm{SH} 2$ antiserum (used at a 1:4000 dilution), as well as all polyclonal antibodies, were preadsorbed against wild-type embryos prior to use.

Whole-mount immunostaining of embryos was carried out as described (Ashburner 1989). Primary antibodies were detected with the ABC-elite system (Vector Laboratories). Immunostained embryos were mounted in $90 \%$ glycerol and photographed under DIC optics (Zeiss Axiophot) using Ektachrome 160T film.

Cuticles were prepared essentially as described (Wieschaus and Nüsslein-Volhard 1986) and photographed under phase-contrast microscopy using Ektachrome 160T film. Photographic slides were scanned with a Polaroid Sprintscan 35 scanner and processed with Adobe Photoshop 4.0 for Macintosh. Composite figures were prepared with Adobe Illustrator 6.0.

\section{In situ hybridizations}

Third instar larva polytene chromosome squashes were prepared according to Pardue (1986) and probed by in situ hybridization with biotin-labeled DNA fragments (Engels et al. 1986). Whole-mount in situ hybridization of embryos was carried out according to Tautz and Pfeifle (1989) using digoxygenin-labeled RNA probes prepared by in vitro transcription (Boehringer Mannheim).

\section{Molecular characterization of the shark ${ }^{1}$ allele}

shark ${ }^{1}$ mutant flies were rescued in trans to $D f(2 R) I p 7$ by the hs-shark-10 transgene. Genomic DNA was prepared from flies of the genotype $W$; shark ${ }^{1} / D f(2 R) I p 7$; hs-shark-10 and used as template for subsequent PCR procedure. Primers used to amplify from the mutant genome are primer $1,5^{\prime}$-CAAAACGCAGCCAGGATCGGAGCTCATGGCCC-3'; primer 2 , 5'-GAATGGTAACAAGGTGCAGTCGG-3'; primer 3, 5'-CG- 
GTGGCAGGTCAATCTCAATGG-3'; and primer 4, 5'-GCGGGATCCGATCTGTGGGTTCCAGATCGTTAATACG-3'. Primers 1 and 3 were used to amplify the $5^{\prime}$ half of the shark gene; primers 2 and 4 for the $3^{\prime}$ half. Primers 1 and 4 are located in the $5^{\prime}$ and $3^{\prime}$ UTR (shark cDNA used in the rescue construct lacks all UTR sequences), permitting selective amplification of the mutant genomic sequence.

\section{Acknowledgments}

This work was supported by National Institutes of Health grant GM 55293 and the Albert Einstein Cancer Center (grant P3013330) (to E.R.S.) and an NSF grant (to R.S.).

The publication costs of this article were defrayed in part by payment of page charges. This article must therefore be hereby marked "advertisement" in accordance with 18 USC section 1734 solely to indicate this fact.

\section{References}

Ashburner, M. 1989. Drosophila: A laboratory manual, 2nd ed., Cold Spring Harbor Laboratory Press, Cold Spring Harbor, NY.

Campos-Ortega, J.A. and V. Hartenstein. 1985. The embryonic development of Drosophila melanogaster. Springer-Verlag, Berlin, Germany.

Chen, Y., M.J. Riese, M.A. Killinger, and F.M. Hoffmann. 1998. A genetic screen for modifiers of Drosophila decapentaplegic signaling identifies mutations in punt, Mothers against dpp and the BMP-7 homologue, 60A. Development 125: 17591768.

Chou, T.-B. and N. Perrimon. 1996. The autosomal FLP-DFS technique for generating germline mosaics in Drosophila melanogaster. Genetics 144: 1673-1679.

Chou, T.-B., E. Noll, and N. Perrimon. 1993. Autosomal P[ovo $^{\text {D1 }}$ ] dominant female-sterile insertions in Drosophila and their use in generating germ-line chimeras. Development 119: 1359-1369

Driever, W., V. Siegel, and C. Nüsslein-Volhard. 1990. Autonomous determination of anterior structures in the early Drosophila embryo by the bicoid morphogen. Development 109: $811-820$.

Engels, W.R., C.R. Preston, P. Thompson, and W.B. Eggleston. 1986. In situ hybridization to Drosophila salivary chromosomes with biotinylated DNA probes and alkaline phosphatase. Focus 8: 6-8.

Erickson, M.R.S., B.J. Galletta, and S.M. Abmayr. 1997. Drosophila myoblast city encodes a conserved protein that is essential for myoblast fusion, dorsal closure, and cytoskeletal organization. J. Cell Biol. 138: 589-603.

Fehon, R.G., I.A. Dawson, and S. Artavanis-Tsakonas. 1994. A Drosophila homologue of membrane-skeleton protein 4.1 is associated with septate junctions and is encoded by the coracle gene. Development 120: 545-557.

Ferrante, A.W., Jr., R. Reinke, and E.R. Stanley. 1995. Shark, a Src homology 2, ankyrin repeat, tyrosine kinase, is expressed on the apical surfaces of ectodermal epithelia. Proc. Natl. Acad. Sci. 92: 1911-1915.

Glise, B. and S. Noselli. 1997. Coupling of Jun amino-terminal kinase and Decapentaplegic in Drosophila morphogenesis. Genes \& Dev. 11: 1738-1747.

Glise, B., H. Bourbon, and S. Noselli. 1995. hemipterous encodes a novel Drosophila MAP kinase kinase, required for epithelial cell sheet movement. Cell 83: 451-461.
Guan, K.L. and J.E. Dixon. 1991. Eukaryotic proteins expressed in Escherichia coli: An improved thrombin cleavage and purification procedure of fusion proteins with glutathione Stransferase. Anal. Biochem. 192: 262-267.

Harden, N.J., J. Lee, H.-Y. Loh, Y.-M. Ong, I. Tan, T. Leung, E. Masner, and L. Lim. 1996. A Drosophila homolog of the Racand Cdc42-activated serine/threonine kinase PAK is a potential focal adhesion and focal complex protein that colocalizes with dynamic actin structures. Mol. Cell. Biol. 16: $1896-1908$.

Heitzler, P., M. Haenlin, P. Ramain, M. Calleja, and P. Simpson. 1996. A genetic analysis of pannier, a gene necessary for viability of dorsal tissues and bristle positioning in Drosophila. Genetics 143: 1271-1286.

Hudson, J.B., S.D. Podos, K. Keith, S.L. Simpson, and E.L. Ferguson. 1998. The Drosophila Medea gene is required downstream of dpp and encodes a functional homolog of human Smad4. Development 125: 1407-1420.

Kiyokawa, E., Y. Hashimoto, S. Kobayashi, H. Sugimura, T. Kurata, and M. Matsuda. 1998. Activation of Rac1 by a Crk SH3-binding protein, DOCK180. Genes \& Dev. 12:33313336.

Knust, E. 1997. Drosophila morphogenesis: Movements behind the edge. Curr. Biol. 7: R558-R561.

Kyriakis, J.M. and J. Avruch. 1996. Sounding the alarm: Protein kinase cascades activated by stress and inflammation. J. Biol. Chem. 271: 24313-24316.

Leptin, M. 1994. Morphogenesis. Control of epithelial cell shape changes. Curr. Biol. 4: 709-712.

Lewis, E.B. and F. Bacher. 1968. Method of feeding ethyl methane sulfonate (EMS) to Drosophila males. Drosophila Inf. Serv. 43: 193

Lindsley, D.L. and G.G. Zimm. 1992. The genome of Drosophila melanogaster. Academic Press, Inc., San Diego, CA.

Lu, Y. and J. Settleman. 1999. The Drosophila Pkn protein kinase is a Rho/Rac effector target required for dorsal closure during embryogenesis. Genes \& Dev. 13:11681180.

Martinez-Arias, A. 1993. Development and patterning of the larva epidermis of Drosophila. In The development of Drosophila melanogaster (ed. M. Bate and A. Martinez-Arias), pp. 517-607. Cold Spring Harbor Laboratory Press, Cold Spring Harbor, NY.

Nolan, K.M., K. Barrett, Y. Lu, K.-Q. Hu, S. Vincent, and J. Settleman. 1998. Myoblast city, the Drosophila homolog of DOCK180/CED-5, is required in a Rac signaling pathway utilized for multiple developmental processes. Genes \& Dev. 12: $3337-3342$.

Noselli, S. 1998. JNK signaling and morphogenesis in Drosophila. Trends Genet. 14: 33-38.

Noselli, S. and F. Agnes. 1999. Roles of the JNK signaling pathway in Drosophila morphogenesis. Curr. Opin. Genet. Dev. 9: 466-472.

Pardue, M.L. 1986. In situ hybridiztion to DNA of chromosomes and nuclei. In Drosopholia, a practical approach (ed. D.B. Roberts), IRL Press, Oxford, UK.

Raftery, L.A. and D.J. Sutherland. 1999. TGF- $\beta$ family signal transduction in Drosophila development: From Mad to Smads. Dev. Biol. 210: 251-268.

Raftery, L.A., V. Twombly, K. Wharton, and W.M. Gelbart. 1995. Genetic screens to identify elements of the decapentaplegic signaling pathway in Drosophila. Genetics 139: 241-254.

Riesgo-Escovar, J.R. and E. Hafen. 1997a. Common and distinct roles of DFos and DJun during Drosophila development. Science 278: 669-672. 
- 1997b. Drosophila Jun kinase regulates expression of decapentaplegic via the ETS-domain protein Aop and AP-1 transcription factor DJun during dorsal closure. Genes \& Dev. 11: 1717-1727.

Riesgo-Escovar, J.R., M. Jenni, A. Fritz, and E. Hafen. 1996. The Drosophila Jun-N-terminal kinase is required for cell morphogenesis but not for DJun-dependent cell fate specification in the eye. Genes \& Dev. 10: 2759-2768.

Sluss, H.K., Z. Han, T. Barrett, R.J. Davis, and Y.T. Ip. 1996. A JNK signal transduction pathway that mediates morphogenesis and an immune response in Drosophila. Genes \& Dev. 10: $2745-2758$.

Smith, D.B. and K.S. Johnson. 1988. Single-step purification of polypeptides expressed in Escherichia coli as fusions with glutathione S-transferase. Gene 67: 31-40.

Spradling, A.C. and G.M. Rubin. 1982. Transposition of cloned P-elements into Drosophila germline chromosomes. Science 218: 341-347.

Su, Y.-C., J.E. Treisman, and E.Y. Skolnik. 1998. The Drosophila Ste20-related kinase misshapen is required for embryonic dorsal closure and acts through a JNK MAPK module on an evolutionarily conserved signaling pathway. Genes \& Dev. 12: 2371-2380.

Tateno, M., Y. Nishida, and T. Adachi-Yamada. 2000. Regulation of JNK by Src during Drosophila development. Science 287: 324-327.

Tautz, D. and C. Pfeifle. 1989. A non-radioactive in situ hybridization method for the localization of specific RNAs in Drosophila embryos reveals translational control of the segmentation gene hunchback. Chromosoma 98: 81-95.

Terracol, R. and J.A. Lengyel. 1994. The thick veins gene of Drosophila is required for dorsoventral polarity of the embryo. Genetics 138: 165-178.

Thummel, C.S., A.M. Boulet, and H.D. Lipshitz. 1988. Vectors for Drosophila P-element-mediated transformation and tissue culture transfection. Gene 74: 445-456.

Treier, M., D. Bohman, and M. Mlodzik. 1995. Jun cooperates with the ETS domain protein pointed to induce photoreceptor R7 fate in the Drosophila eye. Cell 83: 753-760.

Wieschaus, E. and C. Nüsslein-Volhard. 1986. Looking at embryos. In Drosophila, a practical approach (ed. D.B. Roberts), pp. 199-227. IRL Press, Oxford, UK.

$\mathrm{Xu}, \mathrm{T}$. and G.M. Rubin. 1993. Analysis of genetic mosaics in developing and adult Drosophila tissues. Development 117: 1223-1237.

Xu, X., Z. Yin, J.B. Hudson, E.L. Ferguson, and M. Frasch. 1998. Smad proteins act in combination with synergistic and antagonistic regulators to target Dpp responses to the Drosophila mesoderm. Genes \& Dev. 12: 2354-2370.

Zeitlinger, J. and D. Bohmann. 1999. Thorax closure in Drosophila: Involvement of Fos and the JNK pathway. Development 126: 3947-3956.

Zeitlinger, J., L. Kockel, F.A. Peverali, D.B. Jackson, M. Mlodzik, and D. Bohmann. 1997. Defective dorsal closure and loss of epidermal decapentaplegic expression in Drosophila fos mutants. EMBO I. 16: 7393-7401. 


\section{Erratum}

Genes \& Development 14: 604-614

The Drosophila Shark tyrosine kinase is required for embryonic dorsal closure

Rafael Fernandez, Fumitaka Takahashi, Zhao Liu, Ruth Steward, David Stein, and E. Richard Stanley

The authors' affiliations were inadvertently listed incorrectly. The correct affiliations are as follows:

\section{Rafael Fernandez, ${ }^{1,4}$ Fumitaka Takahashi, ${ }^{1}$ Zhao Liu, ${ }^{3}$ Ruth Steward, ${ }^{3}$ David Stein,,${ }^{1,2}$ and E. Richard Stanley ${ }^{1,5}$}

Departments of ${ }^{1}$ Developmental and Molecular Biology and ${ }^{2}$ Molecular Genetics, Albert Einstein College of Medicine, The Bronx, New York 10461 USA, ${ }^{3}$ Waksman Institute, Department of Molecular Biology and Biochemistry, Rutgers University, Piscataway, New Jersey 08854 USA

${ }^{4}$ Present address: Howard Hughes Medical Institute, University of Pennsylvania Medical School, 415 Curie Boulevard, CRB 320 , Philadelphia, PA 19104. ${ }^{5}$ Corresponding author.

E-MAIL rstanley@aecom.yu.edu: FAX (718) 430-8567. 


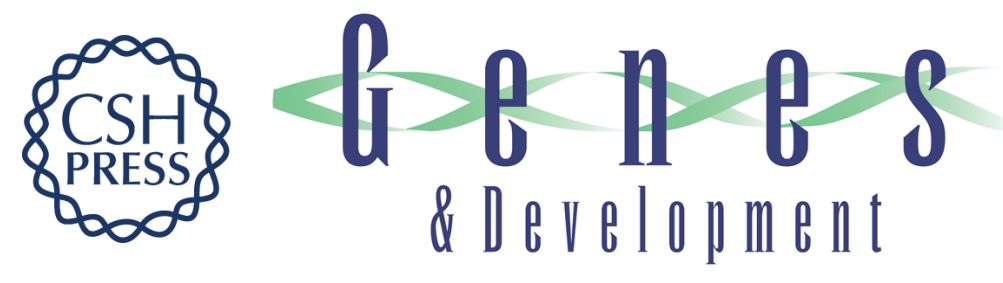

\section{The Drosophila Shark tyrosine kinase is required for embryonic dorsal closure}

Rafael Fernandez, Fumitaka Takahashi, Zhao Liu, et al.

Genes Dev. 2000, 14:

Access the most recent version at doi:10.1101/gad.14.5.604

\section{Related Content Erratum \\ Genes Dev. April , 2000 14: 887}

References This article cites 41 articles, 28 of which can be accessed free at: http://genesdev.cshlp.org/content/14/5/604.full.htmI\#ref-list-1

Articles cited in:

http://genesdev.cshlp.org/content/14/5/604.full.html\#related-urls

\section{License}

Email Alerting

Receive free email alerts when new articles cite this article - sign up in the box at the top Service right corner of the article or click here.

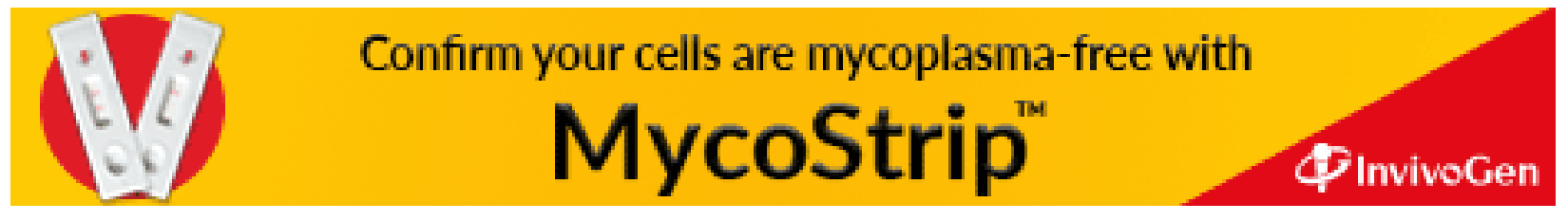

